\title{
Experimental Evidence for Common Driving Effects in Low-Energy Fission from Sublead to Actinides
}

\author{
C. Schmitt $\odot,{ }^{1,}{ }^{*}$ A. Lemasson $\odot,{ }^{2}$ K.-H. Schmidt $\odot,{ }^{3}$ A. Jhingan, ${ }^{4}$ S. Biswas,${ }^{2}$ Y. H. Kim $\odot,{ }^{5}$ D. Ramos $\odot,{ }^{2}$ \\ A. N. Andreyev $\odot,{ }^{6,7,8}$ D. Curien $\odot,{ }^{1}$ M. Ciemala $\odot,{ }^{9}$ E. Clément, ${ }^{2}$ O. Dorvaux,${ }^{1}$ B. De Canditiis, ${ }^{1}$ F. Didierjean, ${ }^{1}$ \\ G. Duchêne, ${ }^{1}$ J. Dudouet $\odot,{ }^{10,11}$ J. Frankland $\odot,{ }^{2}$ B. Jacquot $\odot,{ }^{2}$ C. Raison, ${ }^{6}$ D. Ralet, ${ }^{2}$ B.-M. Retailleau, ${ }^{2}$ \\ L. Stuttgé, ${ }^{1}$ and I. Tsekhanovich ${ }^{12}$ \\ ${ }^{1}$ Institut Pluridisciplinaire Hubert Curien, CNRS/IN2P3-UDS, 67037 Strasbourg Cedex 2, France \\ ${ }^{2}$ GANIL, CEA/DRF-CNRS/IN2P3, BP 55027, 14076 Caen cedex 5, France \\ ${ }^{3}$ Rheinstraße 4, 64390 Erzhausen, Germany \\ ${ }^{4}$ Inter University Accelerator Centre, Aruna Asaf Ali Marg, Post Box 10502, New Delhi 110067, India \\ ${ }^{5}$ Institut Laue-Langevin, 38042 Grenoble Cedex 9, France \\ ${ }^{6}$ Department of Physics, University of York, York YO10 5DD, United Kingdom \\ ${ }^{7}$ Advanced Science Research Center, Japan Atomic Energy Agency, Tokai, Ibaraki 319-1195, Japan \\ ${ }^{8}$ ISOLDE, CERN, CH-1211 Geneve 23, Switzerland \\ ${ }^{9}$ The Niewodniczanski Institute of Nuclear Physics-PAN, 31-342 Kraków, Poland \\ ${ }^{10}$ CSNSM, Université Paris-Sud, CNRS/IN2P3, Université Paris-Saclay, 91405 Orsay, France \\ ${ }^{11}$ Université Lyon, Université Claude Bernard Lyon 1, CNRS/IN2P3, IP2I Lyon, UMR 5822, F-69622 Villeurbanne, France \\ ${ }^{12}$ Université Bordeaux, CNRS, CENBG, UMR 5797, F-33170 Gradignan, France
}

(Received 19 July 2020; revised 22 October 2020; accepted 25 February 2021; published 2 April 2021)

Isotopic distributions of fragments from fission of the neutron-deficient ${ }^{178} \mathrm{Hg}$ nuclide are reported. This experimental observable is obtained for the first time in the region around lead using an innovative approach based on inverse kinematics and the coincidence between the large acceptance magnetic spectrometer VAMOS ++ and a new detection arm close to the target. The average fragment $N / Z$ ratio and prompt neutron $M_{n}$ multiplicity are derived and compared with current knowledge from actinide fission. A striking consistency emerges, revealing the unexpected dominant role of the proton subsystem with atomic number between the $Z=28$ and 50 magic numbers. The origin of nuclear charge polarization in fission and fragment deformation at scission are discussed.

DOI: 10.1103/PhysRevLett.126.132502

In the late 1930s, a new type of disintegration, the socalled fission process, or splitting of a heavy nucleus into two fragments, appeared to be an important finding [1]. It is now well established that the fission process plays a crucial role in fundamental nuclear physics, astrophysics, and societal applications.

Since the discovery of fission, complete fragment mass (A) distributions were measured mainly in the actinide region at low excitation energy ( $E^{*}$ up to a few $\mathrm{MeV}$ above the fission barrier) [2]. For the vast majority, these distributions revealed the favored population of two fragments of very different size (asymmetric fission) as opposed to more balanced splits (symmetric fission). These observations were first traced back to the leading influence of shell-stabilized neutron numbers $(N)$ in the nascent fragments $[3,4]$. Around

Published by the American Physical Society under the terms of the Creative Commons Attribution 4.0 International license. Further distribution of this work must maintain attribution to the author(s) and the published article's title, journal citation, and DOI. the year 2000, progress in experimental methods gave access to high-resolution fragment atomic number $(Z)$ distributions which established instead the leading role of protons in the heavy fragment [5].

Based on the knowledge from actinides, low-energy fission in the preactinide region around lead was expected to be mass symmetric. The measurement [6] of a strong asymmetry in fission of the neutron-deficient ${ }^{180} \mathrm{Hg}$, anticipated to lead to two semimagic ${ }_{40}^{90} \mathrm{Zr}_{50}$ was thus a surprise. Evidence for asymmetric fission was already seen by Itkis et al. for preactinides near to $\beta$ stability [7]. The result on ${ }^{180} \mathrm{Hg}$ triggered an intense experimental [8-18] and theoretical [19-29] worldwide effort in the neutrondeficient lead region. So far, mass and total kinetic energy (TKE) distributions have been reported for about 15 systems. These observables are intimately related to the potential-energy landscape of the fissioning system, which is expected to be governed by the quantum effects in the nascent fragments on the way toward scission. However, it is impossible to ascertain which, among the two partners, and among the two nucleon subsystems, decides on the 

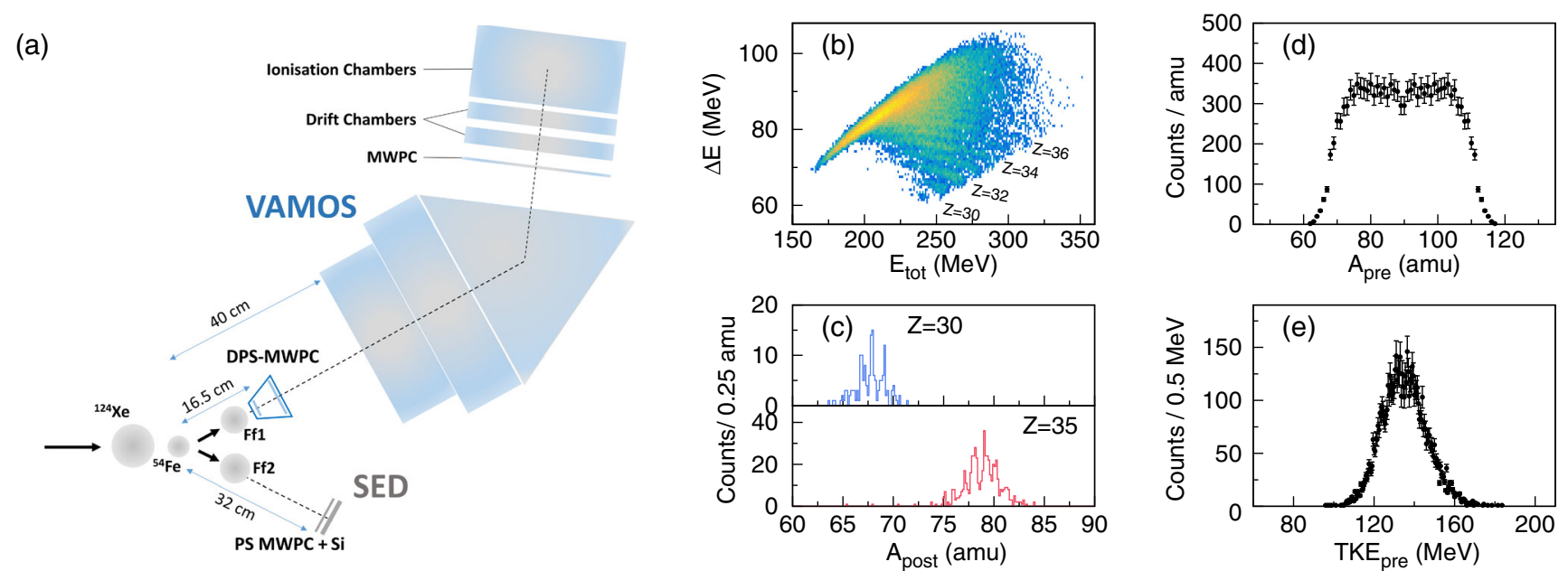

FIG. 1. (a) Schematic of the experimental setup for detecting the coincident fission partners (Ff1 and Ff2). (b) Correlation between the fragment energy loss $\Delta E$ and total energy $E_{\text {tot }}=\Delta E+E_{\text {res }}$ detected in VAMOS ++ . Some $Z$ lines are indicated for reference. (c) Postneutron $A$ distributions for selected $Z$ values. Preneutron (d) $A$ and (e) TKE distributions.

mass partition, as the potential energy results from the combined effect of the protons and neutrons of both fragments. While the $A$ and TKE data confirm the prediction [22] of an island of asymmetric fission in the neutron-deficient region around lead, information on $Z$ and new observables is definitively required to understand what drives asymmetric fission across the nuclear chart.

The fragment neutron-to-proton ratio $N / Z$, a measure of neutron richness, is obtained from the simultaneous identification of fragments $A$ and $Z$. It provides information on the nuclear charge polarization [2] which is the deviation from the unchanged-charge-density (UCD) assumption [30] where the charge density of the fissioning nucleus is maintained in the primary fragments. While the $N / Z$ may help to discriminate between the influence of each nucleon type, neutron richness in one fragment remains necessarily correlated with neutron deficiency in the other, preventing the disentangling of the respective role of each fragment. Another interesting observable is the number of neutrons evaporated by a fragment promptly after scission $\left(M_{n}\right)$ which is related to its excitation energy. In low-energy fission, this energy is mainly contributed by the deformation energy at scission, which transforms into intrinsic excitation of the fragment along relaxation to the groundstate shape. An additional amount can arise from an increase in the initial excitation energy depending on the reaction inducing fission. Provided that $E^{*}$ is not too large, $M_{n}$ is thus a measure of the deformation of the emitter right after the split, i.e., it is indicative of the scission configuration and, in turn, of the fission path. Though, both neutrons and protons contribute to the binding energy. Therefore, discriminating between the role of a specific nucleon type of a specific fragment requires combining several observables [2].

So far, for neutron-deficient preactinides, there are no experimental data on either the neutron and proton fragment content, or postscission evaporation. In this Letter, the first measurement of fission-fragment isotopic distributions is reported giving access to the $\langle N\rangle / Z$ ratio and $\left\langle M_{n}\right\rangle$ multiplicity.

The experiment was performed at GANIL, where fission of the neutron-deficient ${ }^{178} \mathrm{Hg}$ compound nucleus was induced at $E^{*}=34 \mathrm{MeV}$ by fusion (time-dependentHartree-Fock calculations [31] predict that possible contamination from quasifission is negligible) with a ${ }^{124} \mathrm{Xe}$ beam $\left(4.3 \mathrm{MeV} / u\right.$ ) impinging on a $130 \mu \mathrm{g} / \mathrm{cm}^{2}$ thick ${ }^{54} \mathrm{Fe}$ target. Fragment $(A, Z)$ identification was achieved owing to the use of inverse kinematics in combination with the VAMOS ++ spectrometer [32]. A schematic view of the setup is shown in Fig. 1(a). VAMOS ++ was placed at $29^{\circ}$ with respect to the beam axis. The velocity vector and $(A, Z)$ identification of one of the fragments were obtained from time-of-flight (TOF), position coordinates $(x, y)$ measured at the entrance and the focal plane of VAMOS ++ and the energy loss and residual energy measured in the ionization chamber at the focal plane. Details about the spectrometer and analysis can be found in Refs. [32,33]. A new second detection (SED) arm consisting of a position-sensitive MWPC (PS-MWPC) backed with a $\mathrm{Si}$ detector [34] was placed at $-35^{\circ}$ at a distance of $\approx 30 \mathrm{~cm}$ from the target to detect the fission partner. The PS MWPC provided the TOF (with respect to VAMOS ++ ), and $(x, y)$ coordinates, while the fragment residual energy was measured in the $\mathrm{Si}$. Calibration of the detectors was made using elastically scattered ${ }^{54} \mathrm{Fe}$ and ${ }^{124} \mathrm{Xe}$ nuclei.

Because of the low fissility of $\mathrm{Hg}$, proper background rejection is crucial. Fission was selected according to gates on the fragment folding angle and on the interdependence of the fragment TOF's expected for two-body kinematics [35] made newly available with the implementation of the SED (see Supplemental Material [36]). Identification of the fragment $Z$ is based on the correlation between the energy 
loss $\Delta E$ and the total energy $E_{\text {tot }}$ from the ionization chamber, see Fig. 1(b) where each line corresponds to a different $Z$. Because of the low kinetic energy of the fission products, the atomic number could be assigned for the light fragments only, up to $Z=38$. Fragment mass could be fully resolved $(\triangle A / A \approx 0.8 \%)$ by VAMOS ++ for all fragments [32]. Figure 1(c) shows the resulting distributions for two representative elements. These isotopic distributions have been corrected for the acceptance of the spectrometer based on the method discussed in Refs. [40,41].

The physics of the fission process is best studied with preneutron emission observables which reflect the situation at the moment of scission, i.e., prior postscission evaporation. As the fragments are physically detected several tens of ns after the reaction, they correspond to the postneutron products. Thanks to the enhancement of VAMOS ++ with the SED arm [36], the kinematical coincidence (so-called $2 v$ ) method [35], which exploits conservation of momentum between the two fragments when the system splits, could be applied to deduce the preneutron $A$ and TKE distributions for the first time at $\operatorname{VAMOS}++$. The preneutron mass resolution amounts to $\approx 4$ amu. Its distribution, see Fig. 1(d), is seen to exhibit a broad shape with a shallow dip at symmetry. This distribution is very similar to those measured at comparable $E^{*}$ for the close-by ${ }^{180} \mathrm{Hg}$ [12] and ${ }^{178} \mathrm{Pt}[16]$ systems. The preneutron TKE distribution, see Fig. 1(e), is Gaussian-like in shape with a mean value of $135(5) \mathrm{MeV}$ and a width $\sigma_{\mathrm{TKE}}=11.1 \mathrm{MeV}$, compatible with Refs. $[12,16]$. The existence of an asymmetric fission mode for ${ }^{178} \mathrm{Hg}$ [8] is thus confirmed. An extended comparison will be presented in Ref. [34]. We focus below on the features specific to the present measurement and going beyond current knowledge.

The experimental postneutron isotopic distributions [Fig. 1(c)] give access to the average postneutron $\langle N\rangle / Z$ as a function of atomic number, shown in Fig. 2(a). The $Z$-identified light fragments are observed to be more neutron rich than the compound nucleus. Since neutron emission decreases the $N / Z$, the preneutron $\langle N\rangle / Z$ would correspond to even larger neutron richness; this is illustrated by the shaded left-hand-side band assuming up to three neutrons evaporated after scission [corresponding to a typical maximum value [2], see also Fig. 2(b)]. Because of conservation laws, the heavy fragment preneutron $\langle N\rangle / Z$ is symmetric about the compound nucleus $N / Z$ of 1.225 , see shaded right-hand-side band. Such a variation of the preneutron $\langle N\rangle / Z$ with fragment size is opposite to that observed in low-energy fission of actinides $[2,42,43]$ where the heavy fragment is more neutron rich. Also, it is contrary to the expectation from a macroscopic picture [4], shown by the green thick dashed line in Fig. 2(a). The average number of neutrons $\left\langle M_{n}\right\rangle$ evaporated per fragment could be determined additionally and for the first time in the neutron-deficient preactinide region. Combining the
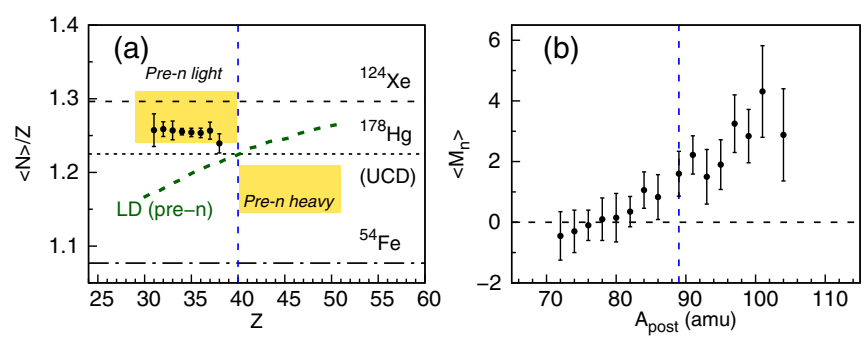

FIG. 2. (a) Postneutron $\langle N\rangle / Z$ as a function of $Z$. The uncertainty is dominated by statistics, with a remainder due to systematics errors ( $Z$ gating and account of acceptance). Shaded yellow bands delimit the regions of the preneutron $\langle N\rangle / Z$, with the green thick dashed line corresponding to the macroscopic prediction. (b) $\left\langle M_{n}\right\rangle$ multiplicity as a function of postneutron mass. The error bars are mainly contributed by statistics that dominates the determination of $\left\langle A_{\text {pre }}\right\rangle$. Dashed vertical lines locate symmetry.

postneutron mass $\left(A_{\text {post }}\right)$ of the fragment unambiguously identified in the spectrometer, and the average preneutron mass $\left(\left\langle A_{\text {pre }}\right\rangle\right)$ of its partner determined above from the coincidence between VAMOS ++ and the SED, the $\left\langle M_{n}\right\rangle$ quantity follows according to $\left\langle M_{n}\right\rangle=178-A_{\text {post }}-\left\langle A_{\text {pre }}\right\rangle$. This equality assumes that evaporation before scission is negligible (according to statistical-model calculations [44], about $80 \%$ of the fission events correspond to first chance). The so-extracted $\left\langle M_{n}\right\rangle$ values are shown in Fig. 2(b) as a function of $A_{\text {post }}$. It appears that $\left\langle M_{n}\right\rangle$ is essentially compatible with zero for the lightest fragments $\left(A_{\text {post }} \lesssim 80\right)$ and increases steadily with $A_{\text {post }}$. A similar rise of $\left\langle M_{n}\right\rangle$ up to fragment masses around 115 is observed in fission of actinides $[2,45]$.

It follows from the above comparisons that while the preneutron $A$ and TKE are similar to those measured for neighboring systems, the newly reported $\langle N\rangle / Z$ data for ${ }^{178} \mathrm{Hg}$ and their evolution with fragment size differs from the known trend in fission of heavier elements. At the same time, the evolution of the $\left\langle M_{n}\right\rangle$ observable shows some consistency with the pattern seen in actinide fission over the same mass range.

Nuclear charge polarization in fission is here investigated with the fragment $\langle N\rangle / Z$ ratio evaluated at scission [37,38] (an alternative measure is the deviation $Z-Z_{\mathrm{UCD}}$ [46]). The preneutron $\langle N\rangle / Z$ could be obtained in this experiment by correcting the postneutron ratio of Fig. 2(a) with the neutron multiplicity of Fig. 2(b) for each $Z$ value. Once the light-fragment preneutron $\langle N\rangle / Z$ are available, the heavy fragment follows from conservation laws. The result for ${ }^{178} \mathrm{Hg}$ is compared to the preneutron $\langle N\rangle / Z$ extracted for low-energy fission of ${ }^{239} \mathrm{U}$ [37] and ${ }^{240} \mathrm{Pu}$ [38] in Fig. 3(a). It shows that the preneutron $\langle N\rangle / Z$ is distributed around the macroscopic model expectation [4] for all cases. The large gap observed between the neutron-deficient ${ }^{178} \mathrm{Hg}$ and the more neutron-rich ${ }^{239} \mathrm{U}$ and ${ }^{240} \mathrm{Pu}$ is thus a trivial macroscopic effect. Deviations from the macroscopic 


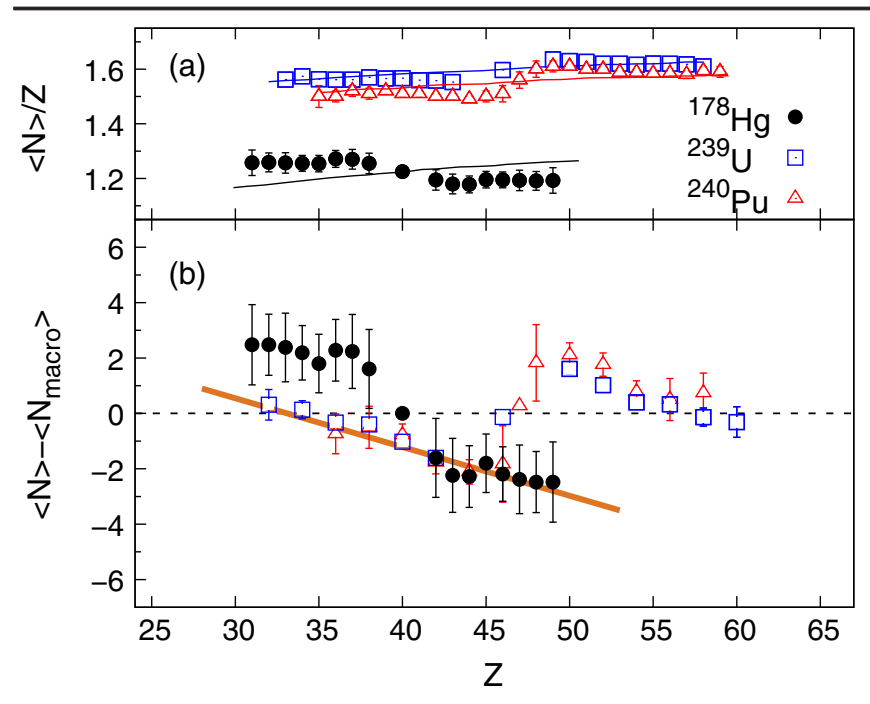

FIG. 3. (a) Preneutron $\langle N\rangle / Z$ for ${ }^{178} \mathrm{Hg}$ (black dots) compared with ${ }^{239} \mathrm{U}$ [37] (blue open squares) and ${ }^{240} \mathrm{Pu}$ [38] (red open triangles). The macroscopic predictions are depicted for each system with a line. (b) $\langle N\rangle-\left\langle N_{\text {macro }}\right\rangle$ difference as a function of $Z$, see text. The downsloping orange line is to guide the eyes.

prediction can be understood as a microscopic (shell effects) contribution to charge polarization. Interestingly, Fig. 3(a) shows that the magnitude of the deviations as a function of $Z$ when moving away from symmetry is similar for the three systems, but of reverse sign for ${ }^{178} \mathrm{Hg}$ compared to ${ }^{239} \mathrm{U}$ and ${ }^{240} \mathrm{Pu}$.

Finding evidence of nuclear structure effects in charge polarization can be difficult, as the macroscopic potential in the $(N-Z)$ direction is rather steep due to the strength of the symmetry energy. Therefore, any shell effect can shift the $N / Z$ only slightly (see, e.g., Ref. [47]). To remove the influence arising from the difference in $N / Z$ of the fissioning system, and isolate nuclear structure effects, the macroscopic contribution was subtracted from the measured $\langle N\rangle / Z$, and the deviation of $\langle N\rangle$ from the macroscopic expectation $\left\langle N_{\text {macro }}\right\rangle$ is shown as a function of $Z$ in Fig. 3(b). A very remarkable observation is that, when sorted according to fragment $Z$, the $\langle N\rangle-\left\langle N_{\text {macro }}\right\rangle$ differences of the light-fragment branch of the actinides and of the heavy-fragment branch of ${ }^{178} \mathrm{Hg}$ define one line. These branches can strictly overlap for $40<Z<46$, only: below $Z=40$ for ${ }^{178} \mathrm{Hg}$ the light partner necessarily lies symmetric about zero, while above $Z=46$ (47) for $\mathrm{U}(\mathrm{Pu})$ symmetric fission sets in. This overlap in the $\langle N\rangle-\left\langle N_{\text {macro }}\right\rangle$ values of the three systems occurs although the corresponding fragments have a very different neutron content, see Fig. 3(a). Therefore, the structural effect behind this observation seems to be highly correlated with the proton subsystem.

Previous measurements established that the heavyfragment peak for actinides fissioning at low energy consists of two independent components [2], which are due to different nascent-fragment shells, and are referred to

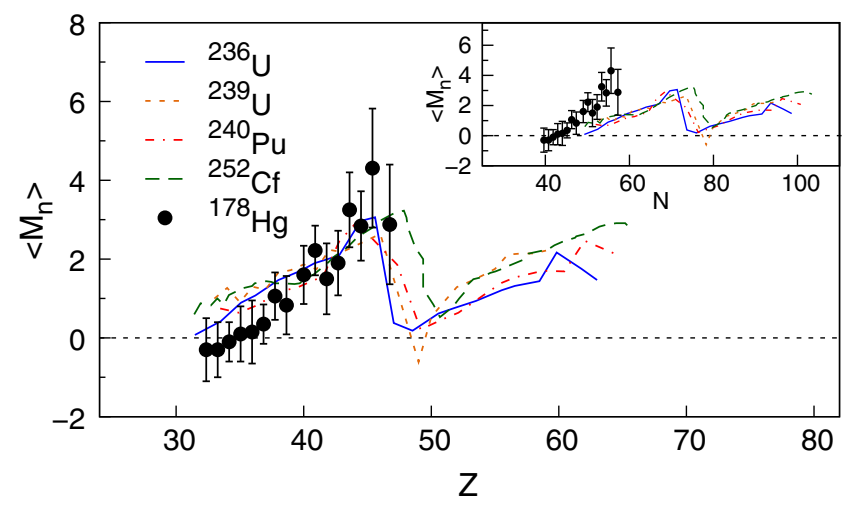

FIG. 4. Neutron $\left\langle M_{n}\right\rangle$ multiplicity as a function of $Z$ for ${ }^{178} \mathrm{Hg}$ (black dots) compared to various actinides (colored lines) $[37,48,49]$. Error bars are not shown for the latter for legibility. The inset shows $\left\langle M_{n}\right\rangle$ as a function of $N$.

as the $S 1$ and $S 2$ modes within Brosa's nomenclature [3], a classification of the fission channels depending on the fragment $N, Z$ and/or shape at scission. Substantial charge polarization in the $S 1$ channel is explained by the concomitant influence of the $N=82$ and $Z=50$ magic numbers [45]. Charge polarization in $S 2$ was very tentatively attributed to the neutron number of the heavy fragment $[2,45]$. The ${ }^{178} \mathrm{Hg}$ data indicate instead that the proton configuration of the light fragment mainly governs charge polarization in the $S 2$ channel in actinide fission. For preactinides, it suggests that polarization is driven by the proton subsystem of the heavy fragment. This work also advocates that it persists up to excitation energy of $30 \mathrm{MeV}$.

To further investigate the role of specific fragments, Fig. 4 compares the average neutron multiplicity per fragment extracted for ${ }^{178} \mathrm{Hg}$ with representative data from low-energy fission of actinides [37,48,49]. Similarly to Fig. 3, $\left\langle M_{n}\right\rangle$ is shown as a function of fragment $Z$ [wherever necessary, measured $A$ values were transformed into $Z$ values using the UCD assumption [30]; note that the deviation of the measured $\langle N\rangle / Z$ 's from UCD observed in Fig. 2(a) does not affect significantly the transformation of the mass axis into the nuclear charge axis]. A striking similarity is found for the values of $\left\langle M_{n}\right\rangle$ in preactinide fission and for the light fragments from actinide fission. This feature is hidden when $\left\langle M_{n}\right\rangle$ is displayed as a function of fragment $N$, see the inset. The overlap of the curves in Fig. 4 is a priori surprising since the larger initial excitation energy in the ${ }^{178} \mathrm{Hg}$ case may have been anticipated to lead to a larger $\left\langle M_{n}\right\rangle$. According to experimental and theoretical arguments [45] the additional initial $E^{*}$ is primarily found in the heavy fragment. The matching of the curves in Fig. 4 thus suggests that, up to $Z$ around $40, M_{n}$ is governed by the deformation of the proton subsystem, since the same $Z$ corresponds to the same $\left\langle M_{n}\right\rangle$ despite a very different neutron content of the fragments in pre- and actinide fission. For $Z \geq 40$, no firm conclusion can be drawn due to the difference in thermal energy between the 
fissioning systems, which primarily affects the excitation energy of the heavy partner as noted above. Though, we note that the larger dissipation of energy between saddle and scission for actinides (due to a longer path) may reduce the difference in the thermal energy available for the fragments above $\mathrm{Zr}$ as compared to preactinides. Taken together with the steady increasing behavior and matching of the curves, it may be speculated that the correspondence between the deformation of the fragments from pre- and actinide fission continues up to $Z=50$.

For low-energy fission of actinides, the position of the asymmetric peaks is primarily given by the shell effects in the heavy fragment $Z$ [5], hence the light-fragment location changes with the fissioning system. This makes it difficult to evidence any structural effects in the light fragment. The present analysis of neutron multiplicities reveals for the first time the role of specific proton configurations in the light fragment in actinide fission.

The interpretation based on deformation driven by the proton subsystem is consistent with the predictions of a "valley" of strong shell effects between the spherical $Z=28$ and 50 magic numbers, correlated with an increasingly large quadrupole deformation [4,50]. According to these calculations, the elongation at scission for the fragments with $Z$ up to $\approx 35$ corresponds to $\beta \approx 0.25-0.35$, a value very similar to their ground-state deformation [51]. Only a small amount of deformation energy is thus available for intrinsic excitation after relaxation. In contrast, much more elongated shapes, up to hyperdeformed, are predicted $[4,50]$ to be stabilized for larger $Z$. These shapes substantially differ from the ground-state shapes, implying a large gain of intrinsic excitation energy when the fragment relaxes. Such large elongations do not appear in the ground state of any nucleus, but can be probed by fission. The consistency in $\left\langle M_{n}\right\rangle$ over a wide range in $N$ seen in Fig. 4 would thus stand for a proof that very elongated shell-stabilized configurations driven by protons indeed exist. Previous data on $\left\langle M_{n}\right\rangle[52,53]$ support the present interpretation, but neutron and proton effects could not be disentangled there. The present combination of a neutrondeficient fissioning nuclide and $Z$ identification was essential to that aim.

The neutron and proton content of the fragments produced in fission in the sublead region was addressed for the first time. The fragment preneutron $\langle N\rangle / Z$ ratio and postscission neutron multiplicity $\left\langle M_{n}\right\rangle$ of ${ }^{178} \mathrm{Hg}$ fissioning at moderate excitation energy were derived from the VAMOS ++ spectrometer at GANIL supplemented with a new detection arm. The measurement of fully resolved fragment mass and atomic number combined with a pertinent sorting of the data according to $Z$ reveals, in a model-independent way, a striking consistency between pre- and actinide fission. The $\langle N\rangle / Z$ ratio shows that nuclear charge polarization in low-energy fission of actinides is dominantly driven by the proton subsystem of the light fragment, in contrast to the fragment yields known to be determined by the heavy partner. The $\left\langle M_{n}\right\rangle$ multiplicity strikingly correlates with shell stabilized, up to very elongated, proton configurations. Both the new observables strongly suggest the crucial role played by the fragments with $Z$ between the 28 and 50 magic numbers over a wide domain of the nuclear chart, challenging current theories [54]. It seems to indicate that Coulomb repulsion tends to permit the restoration of the shell structure of the protons by pushing them back to the nascent fragments, thereby weakening the manifestation of neutron structural effects.

We acknowledge the important technical contributions of J. Goupil, G. Fremont, C. Spitaels, and the excellent support of the GANIL staff. We are grateful to M. Rejmund for help at various stages of the work. P. Möller, A. Navin, C. Simenel, and G. Scamps are acknowledged for critical reading of the manuscript. G. N. Knyazheva and M. Caamaño are thanked for fruitful discussions. The work was partly sponsored by the FrenchGerman collaboration No. 04-48 between IN2P3/CNRSDSM/CEA and GSI, the LIA France-India agreement, and by STFC (U.K.).

Note added in the proof.-In the course of this work, new experimental data in the sublead region appeared $[55,56]$ and which point to the possible driving role of specific fragment proton numbers. Additionnally, Mahata et al. [57] performed an empirical systematics, concluding to a dominant role of the proton subsystem. A smooth evolution between the preactinide and actinide region is also proposed.

*christelle.schmitt@iphc.cnrs.fr

[1] L. Meitner and O. Frisch, Nature (London) 143, 239 (1939), and references therein.

[2] A. N. Andreyev, K. Nishio, and K.-H. Schmidt, Rep. Prog. Phys. 81, 016301 (2018).

[3] U. Brosa, S. Grossmann, and A. Müller, Phys. Rep. 197, 167 (1990).

[4] B. D. Wilkins, E. P. Steinberg, and Chasman, Phys. Rev. C 14, 1832 (1976).

[5] K.-H. Schmidt et al., Nucl. Phys. A665, 221 (2000).

[6] A. N. Andreyev et al., Phys. Rev. Lett. 105, 252502 (2010).

[7] M. G. Itkis et al., Sov. J. Nucl. Phys. 53, 757 (1991) and references therein.

[8] V. Liberati et al., Phys. Rev. C 88, 044322 (2013).

[9] L. Ghys et al., Phys. Rev. C 90, 041301(R) (2014).

[10] G. Boutoux et al., Phys. Procedia 47, 166 (2013).

[11] T. Gorbinet et al., Phys. Procedia 64, 101 (2015).

[12] K. Nishio et al., Phys. Lett. B 748, 89 (2015).

[13] E. Prasad et al., Phys. Rev. C 91, 064605 (2015).

[14] R. Tripathi et al., Phys. Rev. C 92, 024610 (2015).

[15] A. Sen et al., Phys. Rev. C 96, 064609 (2017).

[16] I. Tsekhanovich et al., Phys. Lett. B 790, 583 (2019).

[17] S. Gupta et al., Phys. Rev. C 100, 064608 (2019). 
[18] S. Gupta et al., Phys. Lett. B 803, 135297 (2020).

[19] T. Ichikawa, A. Iwamoto, P. Moller, and A. J. Sierk, Phys. Rev. C 86, 024610 (2012).

[20] S. Panebianco, J. L. Sida, H. Goutte, J. F. Lemaitre, N. Dubray, and S. Hilaire, Phys. Rev. C 86, 064601 (2012).

[21] M. Warda, A. Staszczak, and W. Nazarewicz, Phys. Rev. C 86, 024601 (2012).

[22] P. Möller, J. Randrup, and A. J. Sierk, Phys. Rev. C 85, 024306 (2012); 91, 044316 (2015).

[23] A. V. Andreev, G. G. Adamian, N. V. Antonenko, and A. N. Andreyev, Phys. Rev. C 88, 047604 (2013).

[24] A. V. Andreev, G. G. Adamian, and N. V. Antonenko, Phys. Rev. C 93, 034620 (2016).

[25] J. D. McDonnell, W. Nazarewicz, J. A. Sheikh, A. Staszczak, and M. Warda, Phys. Rev. C 90, 021302(R) (2014).

[26] T. Ichikawa and P. Möller, Phys. Lett. B 789, 679 (2019).

[27] G. Scamps and C. Simenel, Phys. Rev. C 100, 041602(R) (2019).

[28] H. Paşca, A. V. Andreev, G. G. Adamian, and N. V. Antonenko, Phys. Rev. C 101, 064604 (2020).

[29] K. Pomorski, A. Dobrowolski, R. Han, B. Nerlo-Pomorska, M. Warda, Z. Xiao, Y. Chen, L. Liu, and J. L. Tian, Phys. Rev. C 101, 064602 (2020).

[30] R. Vandebosch and J. R. Huizenga, Nuclear Fission (Academic, New York, 1973).

[31] C. Simenel (private communication).

[32] M. Rejmund et al., Nucl. Instrum. Methods Phys. Res., Sect. A 646, 184 (2011).

[33] Y. H. Kim et al., Eur. Phys. J. A 53, 162 (2017).

[34] A. Jhingan et al. (to be published).

[35] D. Hinde, D. Hilscher, H. Rossner, B. Gebauer, M. Lehmann, and M. Wilpert, Phys. Rev. C 45, 1229 (1992).

[36] See Supplemental Material at http://link.aps.org/ supplemental/10.1103/PhysRevLett.126.132502 for details and gain provided by the implementation of the SED, which includes also Refs. [37-39].
[37] D. Ramos et al., Phys. Rev. C 101, 034609 (2020).

[38] M. Caamaño et al., Phys. Rev. C 92, 034606 (2015).

[39] A. Navin et al., Phys. Lett. B 728, 136 (2014).

[40] M. Caamaño et al., Phys. Rev. C 88, 024605 (2013).

[41] D. Ramos et al., Phys. Rev. C 97, 054612 (2018).

[42] E. Pellereau et al., Phys. Rev. C 95, 054603 (2017).

[43] D. Ramos et al., Phys. Rev. C 99, 024615 (2019).

[44] Various evaporation codes were used to estimate the first chance fission including the Nuclear Reaction Video (http:// nrv.jinr.ru/nrv/) and GEF code [K.-H. Schmidt et al., Nucl. Data Sheets 131, 107 (2016)].

[45] K.-H. Schmidt and B. Jurado, Rep. Prog. Phys. 81, 106301 (2018).

[46] U. Quade et al., Nucl. Phys. A487, 1 (1988) and references therein.

[47] P. Möller and T. Ichikawa, Eur. Phys. J. A 51, 173 (2015).

[48] A. S. Vorobyev et al., AIP Conf. Proc. 769, 613 (2005).

[49] O. A. Batenkov et al., AIP Conf. Proc. 769, 1003 (2005).

[50] I. Ragnarsson and R. K. Sheline, Phys. Scr. 29, 385 (1984).

[51] https://www.nndc.bnl.gov/nudat2/.

[52] F. Plasil, R. L. Ferguson, F. Pleasonton, and H. W. Schmitt, Phys. Rev. C 7, 1186 (1973) and references therein.

[53] M. Caamaño and F. Farget, Phys. Lett. B 770, 72 (2017).

[54] K.-H. Schmidt et al. (to be published).

[55] E.Prasad et al., Phys. Lett. B 811, 135941 (2020).

[56] B. M. A. Swinton-Bland, M. A. Stoyer, A. C. Berriman, D. J. Hinde, C. Simenel, J. Buete, T. Tanaka, K. Banerjee, L. T. Bezzina, I. P. Carter, K. J. Cook, M. Dasgupta, D. Y. Jeung, C. Sengupta, E. C. Simpson, and K. Vo-Phuoc, Phys. Rev. C 102, 054611 (2020).

[57] K. Mahata, C. Schmitt, S. Gupta, A. Shrivastava, G. Scamps, and K.-H. Schmidt, arXiv:2007.16184 